\title{
Indicadores antropométricos como preditores na determinação da fragilidade em idosos
}

\author{
Anthropometric indicators as predictors \\ in determining frailty in elderly people
}

Lucas Silveira Sampaio ${ }^{1}$

José Ailton Oliveira Carneiro ${ }^{1}$

Raildo da Silva Coqueiro ${ }^{1}$

Marcos Henrique Fernandes ${ }^{1}$

${ }^{1}$ Universidade Estadual do Sudoeste da Bahia. R. José Moreira Sobrinho $\mathrm{s} / \mathrm{n}$, Jequiezinho. 45206190 Jequié BA Brasil, lucaosampaio@hotmail.com

\begin{abstract}
The scope of this article is to analyze anthropometric indicators as predictors in determining frailty in elderly people. This is a cross-sectional, family-based and analytical study. The survey was composed of 316 elderly subjects. The association between the anthropometric markers and frailty was tested using the Logistic Regression technique. However, the power of frailty diagnosis using anthropometric markers and the identification of the best cutoff points were evaluated using the parameters provided by the Receiver Operating Characteristic (ROC) curve. The anthropometric indicators of nutritional status: calf circumference, body mass index and corrected arm-muscle area are inversely associated with frailty. Thus, an increase in the values of these indicators is a protective factor against this syndrome. Analyzing body mass index and calf circumference simultaneously in order to perform a simpler screening for frail elderly people is recommended, as these indicators show good sensitivity and are easier to measure.
\end{abstract}

Key words Frail Elderly, Anthropometry, Health of the elderly
Resumo O objetivo deste artigo é analisar os indicadores antropométricos como preditores na determinação da fragilidade em idosos. Trata-se de um estudo transversal de base domiciliar e analítico. A pesquisa foi constituída por 316 idosos. A associação entre os marcadores antropométricos e a fragilidade foi testada por meio da técnica de Regressão Logística. Já o poder de diagnóstico de fragilidade, utilizando os marcadores antropométricos e a identificação dos melhores pontos de corte, foi avaliado por meio dos parâmetros fornecidos pela curva Receiver Operating Characteristic. Os indicadores antropométricos de estado nutricional, Perímetro da Panturrilha, Indice de Massa Corpórea e Área Muscular do Braço Corrigida, são inversamente associados à fragilidade, assim o incremento nos valores desses indicadores é fator de proteção para esta sindrome. Recomenda-se que o Perímetro da Panturrilha e o Índice de Massa Corpórea sejam analisados de forma combinada para realizar de forma ainda mais simples a triagem de idosos frágeis, já que possuem boa sensibilidade e têm maior facilidade de mensuração de seus valores.

Palavras-chave Idoso fragilizado, Antropometria, Saúde do idoso 


\section{Introdução}

Dentre os fatores que impactam sobre os idosos estão os sinais da fragilidade, as quais são descritas na literatura como a redução da capacidade de reserva, levando a complicações como quedas, imobilidade, fraturas, hospitalização e incapacidade $^{1,2}$. São definidas também como um estado progressivo de déficits de múltiplos sistemas fisiológicos, que levam a um aumento da vulnerabilidade e diminuição da capacidade de resistência a agentes estressores ${ }^{3-5}$. Destaca-se que os idosos frágeis são mais propensos a consequências como quedas, infecções, deficiência, hospitalização, institucionalização e morte, gerando um grande impacto social e econômico para o governo ${ }^{6}$.

Observa-se, contudo, que apesar de haver fenótipos que identificam indivíduos frágeis, como o proposto por Fried et al. ${ }^{4}$, baseado em cinco critérios (perda de peso, fraqueza muscular, baixa resistência e energia, diminuição da velocidade de marcha e baixo nível de atividade física), ainda são escassos os estudos sobre preditores de fragilidade de fácil execução, como, por exemplo, um indicador simples capaz de realizar a triagem de indívíduos frágeis antes da aplicação desses critérios.

Estudos demonstram que há relação entre a redução da capacidade física e alterações antropométricas ${ }^{7}$. Neste contexto, indicadores antropométricos como Índice de Massa Corpórea $(\mathrm{IMC})^{8,9}$, Peso ${ }^{10}$, Relação Cintura-Quadril $(\mathrm{RCQ})^{11,12}$, Perímetro Braquial $(\mathrm{PB})^{13}$, Perímetro da Panturrilha (PP $)^{14}$ e Circunferência da Cintu$\mathrm{ra}^{15}$ são apontados como possíveis indicadores de alterações no estado nutricional e/ou capacidade física e/ou fragilidade.

A antropometria trata-se de um método não invasivo utilizado para avaliar o tamanho, as proporções e a composição do corpo humano, refletindo tanto a saúde como o estado nutricional, e prevendo o desempenho, estado de saúde e sobrevivência de indivíduos ${ }^{16,17}$. Dentre as principais vantagens desse método estão a operacionalização simples e de baixo custo, facilitando, dentre outras coisas, o treinamento dos profissionais e a utilização deste recurso em locais onde não são possíveis operacionalizar métodos mais sofisticados, como em pesquisas domiciliares e em regiões com menor desenvolvimento econômico.

Além disso, para a World Health Organization $^{17}$, a utilização dos indicadores antropométricos pode contribuir na prática clínica e na avaliação epidemiológica. Neste sentido, identificar um indicador antropométrico capaz de realizar a triagem da fragilidade poderá contribuir na prática clínica para identificação e prevenção precoce da síndrome.

Deste modo, o objetivo deste estudo foi avaliar a associação de indicadores antroprométricos de estado nutricional com a fragilidade em idosos residentes em comunidade, além de identificar qual dos indicadores melhor discrimina esta síndrome.

\section{Métodos}

Trata-se de um estudo com delineamento transversal e analítico, elaborado com dados de uma pesquisa de base populacional e domiciliar denominada "Estado nutricional, comportamentos de risco e condições de saúde dos idosos de Lafaiete Coutinho-BA", o qual foi aprovado pelo Comitê de Ética em Pesquisa da Universidade Estadual do Sudoeste da Bahia. Todos os participantes assinaram um termo de consentimento livre e esclarecido, concordando em participar da pesquisa.

A população do estudo foi composta de 355 idosos. Destes, 17 se recusaram a participar e houve 22 perdas de idosos que não foram localizados após três visitas domiciliares. Desta forma, dos 355 idosos que compunham a população do estudo, 316 (89\%) participaram da pesquisa. A localização das residências foi obtida por meio dos registros da Estratégia de Saúde da Família.

A coleta dos dados aconteceu no mês de janeiro de 2011, após autorização e apoio da Secretaria Municipal de Saúde de Lafaiete Coutinho, e foi desenvolvida em duas etapas. A primeira consistiu de uma entrevista domiciliar abrangendo informações pessoais e testes de resistência e locomoção. A segunda etapa foi realizada em duas Unidades de Saúde da Família e incluiu antropometria e teste de força de preensão manual.

A fragilidade foi diagnosticada de acordo com os cinco critérios propostos por Fried et al. ${ }^{4}$ :

Perda de peso não intencional: foi definida por meio do autorrelato da perda de peso não intencional $\geq 3 \mathrm{~kg}$ durante os últimos 12 meses, adaptado por Alvarado et al. ${ }^{18}$;

Fraqueza muscular: foi avaliada a Força de Preensão Manual (FPM), por meio de um dinamômetro hidráulico (Saehan Corporation SH5001, Korea). A fraqueza muscular foi definida de acordo com o sexo e índice de massa corporal $\left[\mathrm{IMC}=\right.$ massa corporal $\left.(\mathrm{kg}) / \operatorname{estatura}^{2}(\mathrm{~m})\right]$. Primeiramente, o IMC foi classificado em três ca- 
tegorias: $<22 \mathrm{~kg} / \mathrm{m}^{2}=$ baixo peso; $22,0 \leq \mathrm{IMC} \leq$ $27 \mathrm{~kg} / \mathrm{m}^{2}=$ adequado; $>27 \mathrm{~kg} / \mathrm{m}^{2}=$ sobrepeso $^{19}$. Em seguida, os pontos de corte para a FPM $(\mathrm{kg})$ foram fixados no percentil 25 para cada categoria de IMC. Dessa forma, foram estabelecidos os seguintes pontos de corte: baixo peso, FPM $\leq 19$ e $11 \mathrm{kgf}$; peso adequado, FPM $\leq 21$ e 15 kgf; sobrepeso, FPM $\leq 22$ e 14 kgf, para homens e mulheres, respectivamente. Os idosos que foram incapazes de realizar o teste devido a limitações físicas foram considerados com fraqueza muscular.

Baixa resistência (fadiga):foi definida com base em duas questões da Geriatric Depression Scale ${ }^{20}$ : Você deixou de lado muitas de suas atividades e interesses? e Você se sente cheio de energia? Uma resposta positiva à primeira pergunta e/ou uma resposta negativa para a segunda foram considerados indícios de baixa resistência e energia.

Lentidão na marcha: foi definida por meio do desempenho físico no teste de caminhada de 2,44 $\mathrm{m}$, em que o indivíduo iniciava sentado em uma cadeira, levantava e realizava o percurso orientado e cronometrado pelo avaliador, assim como descrito e detalhado em estudo prévio ${ }^{21}$. A lentidão foi definida de acordo com o sexo e estatura. Primeiramente, a estatura foi clasificada em duas categorias com base na mediana (percentil 50): $\leq$ mediana (homens $\leq 1,61 \mathrm{~m}$ e mulheres $\leq 1,49$ $\mathrm{m}$ ) e $>$ mediana (homens $>1,61$ m e mulheres $>$ $1,49 \mathrm{~m})$. Em seguida, os pontos de corte do tempo gasto para realizar o teste de caminhada foram fixados no percentil 75, para cada categoria de estatura. Dessa forma, foram estabelecidos os seguintes pontos de corte para lentidão: estatura $\leq$ mediana, $\geq 5 \mathrm{~s} \mathrm{e} \geq 6$ s (para homens e mulheres, respectivamente); e estatura $>$ mediana, $\geq 4$ $\mathrm{s}$ (para ambos os sexos). Os indivíduos que se mostraram incapazes de realizar o teste devido a limitações físicas foram considerados com lentidão.

Baixo nivel de atividade física: $\mathrm{O}$ instrumento utilizado para avaliar o nível de atividade física habitual foi a versão longa do International Physical Activity Questionnaire (IPAQ) ${ }^{22}$, adaptada para idosos ${ }^{23}$. Os idosos que realizaram menos de 150 minutos por semana, em atividades físicas moderadas e/ou vigorosas, foram considerados com baixo nível de atividade física.

Foi criada uma variável ordinal com escores variando de zero a cinco, a partir do somatório dos cinco critérios descritos anteriormente, sendo adotada a seguinte classificação (4):ausência de critérios = não frágil; 1 ou 2 critérios presentes $=$ pré-frágil $; 3$ critérios presentes $=$ frágil. Para termos de análise, a variável fragilidade foi dico- tomizada em frágil ( $\geq 3$ critérios) e não frágil (< 3 critérios).

Para a classificação de fragilidade, foram incluídos apenas os idosos que responderam, no mínimo, quatro dos cinco critérios elegíveis para identificá-lo como frágil, pré-frágil e não frágil.

Os indicadores antropométricos avaliados no presente estudo foram: índice de massa corporal (IMC), perímetro da panturrilha $(\mathrm{PP})$ e área muscular do braço corrigida (AMBc).

A massa corporal (MC) foi medida com balança digital portátil (ZhongshanCamry Eletronic, G-Tech Glass 6, China), com o indivíduo descalço e usando o mínimo de roupas possível. A estatura foi mensurada de acordo com a técnica de Frisancho ${ }^{24}$, utilizando um estadiômetro compacto portátil (Wiso, China), instalado em local adequado, segundo as normas do fabricante, estando o idoso descalço, ereto, com os pés unidos, e calcanhares, nádegas e cabeça em contato com a parede, mantendo os olhos fixos num eixo horizontal paralelo ao chão.

A dobra cutânea triciptal (DCT) foi mensurada com plicômetro (WCS, Brasil) de acordo com Harrison et al. ${ }^{25}$. O perímetro do braço (PB) e perímetro da panturrilha (PP), conforme Callaway et al. ${ }^{26}$ foram avaliados utilizando uma trena antropométrica inelástica $\left(\mathrm{ABN}^{\mathrm{TM}}\right.$, Brasil). O PP foi medido com o indivíduo na posição sentada, sem contração da musculatura colocada no ponto de maior perímetro da perna. O PB foi avaliado no ponto medial entre o acrômio e o olécrano, sendo o braço posicionado ao lado do corpo, de modo relaxado.

Foram calculados o IMC [massa corporal $(\mathrm{kg})$ /estatura $\left.^{2}(\mathrm{~m})\right]$ e a $\mathrm{AMBc}^{27}\{[$ (perímetro do braço - ๘ $\mathrm{x}$ DCT $\left.)^{2} / 4 \times \varpi\right]$ - 10, para homens; [(perímetro do braço - ๘ x DCT $\left.)^{2} / 4 \times \varpi\right]-6,5$, para mulheres\}.

As variáveis de ajuste foram: grupo etário (60-69, 70-79 e $\geq 80$ anos); sexo; hospitalização nos últimos 12 meses ( de saúde [positiva (boa, muito boa e excelente) e negativa (regular e má)]; e capacidade funcional. Estas são variáveis que se associam com fragilidade e podem ser potenciais fatores de confusão ${ }^{28}$.

A capacidade funcional foi avaliada por meio das informações acerca das atividades básicas da vida diária (ABVD), através da Escala de $\mathrm{Katz}^{29}$, e das atividades instrumentais da vida diária (AIVD), através da Escala de Lawton ${ }^{30}$. Foi construída uma escala de incapacidade funcional hierárquica ${ }^{31}$ caracterizando três categorias: independentes, dependentes nas AIVD, dependentes nas ABVD e AIVD. 
Para a análise descritiva das características da população, foram calculadas as frequências absolutas, médias e desvios padrão. A associação entre os indicadores antropométricos (variáveis independentes) e a fragilidade (variável dependente) foi testada por meio da técnica de Regressão Logística. Foram calculados modelos robustos ajustados para estimar as odds ratio (OR), com os seus respectivos intervalos de confiança de 95\% (IC95\%). O poder de diagnóstico de fragilidade pelos indicadores antropométricos e a identificação dos melhores pontos de corte foram avaliados por meio dos parâmetros fornecidos pela curva Receiver Operating Characteristic (ROC):área sob a curva ROC (ACR), sensibilidade e especificidade.

Em todas as análises o nível de significância adotado foi de $5 \%(\alpha=0,05)$. Os dados foram analisados no The Statistical Package for Social Sciences para Windows (SPSS 22.0, 2013, SPSS, Inc, Chicago, IL) e MedCalc (versão 9.1.0.1, 2006).

\section{Resultados}

Participaram da pesquisa 316 idosos, sendo $54,2 \%$ do sexo feminino. A média de idade da população estudada foi de 74,8 $\pm 9,8$ anos. As demais características descritivas são mostradas nas Tabelas 1 e 2. Foi identificado um maior número de idosos hospitalizados (75,5\%), com percepção de saúde negativa $(58,1 \%)$, e $41,1 \%$ eram dependentes para as atividades instrumentais da vida diária (AIVD). A prevalência de fragilidade foi de $23,8 \%$, destacando-se que a fragilidade pode ser avaliada em 90,5\% dos idosos da população do estudo.

Na Tabela 2 encontram-se as variáveis quantitativas do estudo. Destaca-se uma discrepância entre o perímetro mínimo e máximo da $\mathrm{AMBc}$, e também a grande diferença entre os valores mínimo e máximo do IMC entre os idosos.

Na Tabela 3 é mostrada a associação entre fragilidade e os indicadores antropométricos do estado nutricional. Essas análises foram feitas apenas com os 286 idosos em que foi possível realizar a avaliação da fragilidade pelo fenótipo de Fried et al. ${ }^{4}$. Foi mostrado por meio do modelo de regressão logística que todos os indicadores antropométricos foram inversamente associados à fragilidade $(\mathrm{p}<0,01)$, indicando que $\mathrm{o}$ aumento em uma unidade do IMC, AMBc e PP diminuiu em aproximadamente $10 \%, 7 \%$ e $15 \%$, respectivamente, a probabilidade de fragilidade em idosos.

A comparação das áreas sob a curva ROC entre os indicadores antropométricos do estado nutricional pode ser observada na Figura 1. Os indicadores $\mathrm{AMBc}$, IMC e PP apresentaram o valor da área sob a curva ROC superior a $60 \%$. O valor da área sob curva ROC (ASC) indica que esses indicadores são capazes de discriminar os idosos que apresentaram a fragilidade. Destaca-se que não houve diferença estatística nos percentuais sob a curva ROC entre indicadores utilizados ( $\mathrm{p}$ $>0,05)$.

A Tabela 4 apresenta os valores de sensibilidade e especificidade para os indicadores antropométricos do estado nutricional e seus respectivos pontos de cortes encontrados por meio da curva ROC. Foi mostrado, por meio dos parâmetros da curva ROC, que o IMC foi o indicador que apresentou maior sensibilidade, enquanto que a $\mathrm{AMBc}$ apresentou maior especificidade; entretanto, o IMC foi o marcador que apresentou melhor equilíbrio entre sensibilidade e especificidade, com ambos os valores podendo ser considerados moderados.

Tabela 1. Análise descritiva das variáveis qualitativas do estudo. Lafaiete Coutinho-BA, Brasil, 2011.

\begin{tabular}{lcrc}
\hline \multicolumn{1}{c}{ Variáveis } & $\begin{array}{c}\% \\
\text { resposta }\end{array}$ & N & $\%$ \\
\hline Grupo Etário & 99,7 & & \\
60-69 anos & & 115 & 36,5 \\
$70-79$ anos & & 106 & 33,7 \\
$\geq 80$ anos & 100,0 & & 29,8 \\
Sexo & & 143 & 45,3 \\
$\quad$ Masculino & & 173 & 54,7 \\
Feminino & 99,4 & & \\
Hospitalização & & 237 & 75,5 \\
$\quad$ Sim & & 77 & 24,5 \\
$\quad$ Não & 97,2 & & \\
Capacidade funcional & & 130 & 42,3 \\
$\quad$ Independente & & 51 & 16,6 \\
$\quad$ Dependente ABVD & & 126 & 41,1 \\
$\quad$ Dependente AIVD & & & \\
Auto percepção de saúde & 95,9 & 127 & 41,9 \\
$\quad$ Positiva & & 176 & 58,1 \\
$\quad$ Negativa & & & \\
Fragilidade & & 68 & 23,8 \\
Frágil & 90,5 & & 76,2 \\
$\quad$ Não frágil & & & \\
\hline ABVD, Atividades Básicas da Vida Diária; AIVD, Atividades \\
Instrumentais da Vida Diária. & & &
\end{tabular}


Tabela 2. Análise descritiva das variáveis quantitativas do estudo. Lafaiete Coutinho-BA, Brasil, 2011.

\begin{tabular}{lccrc}
\hline \multicolumn{1}{c}{ Variáveis } & \% resposta & Média & DP & Mín - máx \\
\hline $\mathrm{IMC}\left(\mathrm{kg} / \mathrm{m}^{2}\right)$ & 95,3 & 24,6 & 4,64 & $13,6-41,2$ \\
$\mathrm{AMBc}\left(\mathrm{cm}^{2}\right)$ & 97,2 & 32,9 & 9,1 & $9,72-56,08$ \\
Perímetro da panturrilha $(\mathrm{cm})$ & 97,5 & 33,2 & 3,6 & $22,1-43,0$ \\
\hline
\end{tabular}

DP, desvio padrão; IMC, índice de massa corporal; AMBc, área muscular do braço corrigida; PP, perímetro da panturrilha.

Tabela 3. Associação da fragilidade com os indicadores antropométricos do estado nutricional em idosos. Lafaiete Coutinho-BA, Brasil, 2011.

\begin{tabular}{lccc}
\hline \multicolumn{1}{c}{ Variáveis } & OR $^{*}$ & IC95\% & p-valor \\
\hline $\mathrm{IMC}\left(\mathrm{kg} / \mathrm{m}^{2}\right)$ & 0,90 & $0,83-0,97$ & 0,007 \\
$\mathrm{AMBc}\left(\mathrm{cm}^{2}\right)$ & 0,93 & $0,89-0,97$ & 0,001 \\
Perímetro da panturrilha $(\mathrm{cm})$ & 0,85 & $0,77-0,94$ & 0,002 \\
\hline
\end{tabular}

OR, odds ratio; IMC, índice de massa corporal; AMBc, área muscular do braço corrigida; PP, perímetro da panturrilha. *Ajustada para idade, sexo, hospitalização, capacidade funcional e autopercepção de saúde.

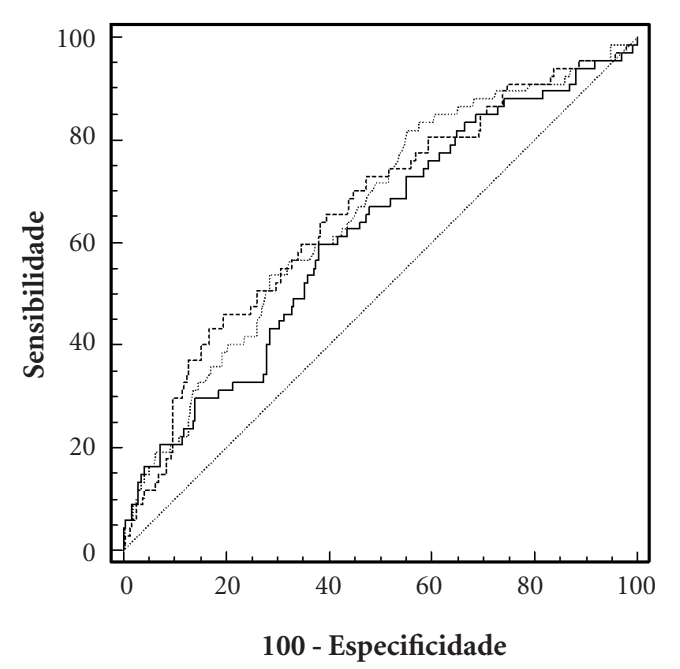
Índice de Massa Corporal $=0,62(0,56-0,68)$
$\ldots \ldots$ Área Muscular do Braço Corrigida $=0,67(0,61-0,72)$
$\ldots \ldots \ldots \ldots \ldots$ Perímetro da Panturrilha $=0,67(0,61-0,72)$

Figura 1. Curvas ROC comparando indicadores antropométricos do estado nutricional utilizados no estudo como discriminadores de fragilidade em idosos. Lafaiete Coutinho-BA, Brasil, 2011.

\section{Discussão}

Este estudo identificou uma associação inversa entre os indicadores antropométricos de estado nutricional PP, IMC e AMBc e a fragilidade, os quais podem ser utilizados como instrumentos de triagem simples e rápida para detectar idosos frágeis.

No presente estudo o IMC foi apontado como o marcador com maior sensibilidade $(59,7 \%)$ para a fragilidade, demonstrando a capacidade de identificar indivíduos frágeis. Além disso, a partir do ponto de corte encontrado, $23,4 \mathrm{~kg} / \mathrm{m}^{2}$, pode-se inferir que indivíduos com valores de IMC abaixo deste valor possuem maior probabilidade de serem frágeis

O resultado encontrado, relacionando baixos valores de IMC com fragilidade, pode ser explicado devido ao IMC sofrer influência de fatores como a sarcopenia durante o processo de envelhecimento. A sarcopenia é caracterizada por perda de massa muscular acompanhada por diminuição de força muscular ${ }^{13,32,33}$, um dos critérios para detecção da fragilidade. Além disso, a fraqueza muscular leva à diminuição do desempenho físico, podendo influenciar diretamente sobre outros critérios diagnósticos dessa síndrome como a lentidão motora e a redução da atividade física. Pierine et al. ${ }^{34}$ apontam ainda que o desuso da musculatura devido a imobilismo e sedentarismo, cuja prevalência é elevada em idosos, também leva à hipotrofia muscular. Entretanto, é importante destacar que a perda de peso não traduz somente a perda muscular, e o aumento de massa gorda pode confundir a avaliação real ${ }^{28,36}$. [falta a citação 35]

Além disso, Reis Junior ${ }^{28}$ sugere que ainda são discutíveis os resultados de estudos que ponderaram a associação do IMC com fragilidade, pois ainda que estudos demonstrem que homens ido- 
Tabela 4. Pontos de corte, sensibilidade e especificidade dos marcadores antropométricos do estado nutricional como discriminadores de fragilidade em idosos. Lafaiete Coutinho-BA, Brasil, 2011.

\begin{tabular}{|c|c|c|c|}
\hline Variáveis & Ponto de corte & Sensibilidade & Especificidade \\
\hline IMC $\left(\mathrm{kg} / \mathrm{m}^{2}\right)$ & 23,4 & 59,7 & 63,6 \\
\hline $\operatorname{AMBc}\left(\mathrm{cm}^{2}\right)$ & 27,4 & 47,1 & 82,1 \\
\hline $\mathrm{PP}(\mathrm{cm})$ & 32,0 & 54,4 & 73,3 \\
\hline
\end{tabular}

IMC, índice de massa corporal; $\mathrm{AMBc}$, área muscular do braço corrigida; PP, perímetro da panturrilha.

sos frágeis possuem valores inferiores de $\mathrm{IMC}^{28,36}$ outros demostram aumento do IMC em indivíduos frágeis ${ }^{37}$.

Em relação à $\mathrm{AMBc}$, foi o marcador antropométrico que apresentou maior capacidade de identificar idosos não frágeis, apresentando uma especificidade de 82,1\%. Assim, indivíduos com valores de $\mathrm{AMBc}$ acima de $27,4 \mathrm{~cm}^{2}$ possuem maior probabilidade de não serem frágeis. Destaca-se que não foram encontrados estudos associando este marcador à fragilidade, dificultando possíveis comparações. Entretanto, a gordura corporal e a massa muscular esquelética (MME) têm sido utilizados como preditores de níveis de saúde durante todo o ciclo de vida. A redução da MME está relacionada ao maior risco de quedas, fratura por fragilidade, infecção e má cicatrização de feridas em idosos ${ }^{38,39}$.

Autores apontam ainda que a redução da massa magra é esperada no processo de envelhecimento normal, no qual ocorrem alterações na coordenação nervosa e, portanto, na ação da musculatura esquelética ${ }^{39,40}$. Assim, a avaliação da $\mathrm{AMBc}$ poderá evidenciar alterações na MME e suas consequências como a sarcopenia e fraqueza muscular, características presente no quadro de fragilidade $^{38}$.

Ainda em relação à $\mathrm{AMBc}$, a DCT reflete a gordura subcutânea, enquanto o perímetro do braço $(\mathrm{PB})$ leva em conta o diâmetro do úmero e dos músculos esqueléticos e gordura que cobrem o membro, demonstrando assim, alterações na massa magra e gordura. É importante ressaltar que estudos apontam haver uma diminuição mais acentuada da massa muscular em membros nos idosos. Destacando-se ainda que as medidas antropométricas do braço, incluindo a DCT e PB podem ser introduzidas facilmente em idosos residentes em comunidades, por ser um método rápido, prático, barato e não invasivo, tornando, desta forma, o cálculo da $\mathrm{AMBc}$ simples a partir desses valores obtidos ${ }^{39}$.
Em relação ao perímetro da panturrilha, identificado neste estudo como um marcador com alta capacidade de realizar a triagem de idosos não frágeis (Especificidade $=73,3 \%$ ), é considerado pela Organização Mundial da Saúde como a mais sensível medida antropométrica de massa muscular em idosos ${ }^{17}$.

Para Ravaglia et al. ${ }^{5}$, o PP é um indicador clínico de massa magra, sendo utilizado para identificar idosos com sarcopenia, uma característica essencial da fragilidade. Além disso, estes mesmos autores, assim como Vellas et al..$^{39}$, também utilizaram o ponto de corte para o PP de $31 \mathrm{~cm}$, valor muito próximo ao encontrado como ponto de corte do presente estudo, $32,0 \mathrm{~cm}$. Nesse contexto, aumentar a circunferência das extremidades, especialmente PP, é demonstrado como fator de proteção da saúde ${ }^{40,41}$, sendo ainda possível inferir a partir do resultado do presente estudo que valores maiores de PP são também fator de prevenção para a fragilidade.

A diminuição nos valores das variáveis indicativas de reserva de massa muscular em idosos é preocupante, pois as alterações musculares acarretam em manifestações clínicas que podem gerar déficit funcional nos idosos e levar a situações como quedas, hospitalização e redução da qualidade de vida ${ }^{41,42}$.

Ao buscar utilizar dados de base populacional com o intuito de estimar valores de ponto de corte para discriminação da fragilidade através dos indicadores antropométricos em comunidade de baixo desenvolvimento econômico-social, este estudo torna-se inovador e importante pois identificou uma possibilidade de triagem de baixo custo e simples operacionalização. Para Jones et al. ${ }^{43}$, além de ser recomendável, é bastante útil usar medidas confiáveis e de fácil aplicação na prática clínica de geriatria. A partir dos resultados do presente estudo, pode-se inferir que é aconselhável a utilização dos indicadores PP, $\mathrm{AMBc}$ e IMC, em conjunto como preditores e 
discriminadores para realizar a triagem de idosos frágeis. Destacando-se que a maior especificidade encontrada na $\mathrm{AMBc}$ faz desse marcador o melhor para realizar, de forma isolada, a triagem de indivíduos que não possuem síndrome.

Uma das limitações deste estudo está relacionada ao seu delineamento transversal, o qual não permite estabelecer relação de causalidade entre mudanças nos indicadores antropométricos e fragilidade. Destaca-se ainda que o PP, assim como o perímetro do braço $(\mathrm{PB})$, reflete a massa muscular do corpo e pode ser influenciado pela alteração de peso. Desta forma, fatores como o processo de envelhecimento, condições de atividade física e nutricionais podem influenciar o tamanho destas medidas antropométricas ${ }^{14}$.

Entretanto, é importante destacar que este estudo permite a valorização e incentivo do uso de indicadores antropométricos de fácil obtenção, baixíssimo custo e não invasivo na prática clínica dos profissionais de saúde com o objetivo de prevenir, manter ou melhorar a monitorização de idosos frágeis.

\section{Considerações finais}

Os indicadores antropométricos de estado nutricional PP, IMC e AMBc apresentaram associação significativa e inversamente proporcional com a síndrome da fragilidade. Recomenda-se, por exemplo, que o IMC e o PP possam ser analisados de forma combinada para realizar de forma ainda mais simples a triagem de idosos frágeis, já que possuem boa sensibilidade e têm maior facilidade de mensuração de seus valores. Deve-se também utilizar a AMBc combinada com o IMC na prática clínica, visando a identificação da fragilidade.

Considerando o baixo custo e facilidade de obtenção de medidas antropométricas, defende-se que esses indicadores antropométricos de estado nutricional, e seus respectivos pontos de corte identificados, podem ser usados para triagem da fragilidade, tornando o diagnóstico mais ágil, contribuindo para as ações de prevenção e promoção da saúde para a população idosa e, assim, favorecendo a atuação de vigilância a saúde.

\section{Colaboradores}

LS Sampaio, JAO Carneiro, RS Coqueiro e MH Fernandes contribuíram de forma igual em todas as etapas de confecção e elaboração do estudo e do artigo. 


\section{Referências}

1. Collard RM, Boter H, Schoevers RA, Voshaar RCO. Prevalence of Frailty in Community Dwelling Older Persons : A Systematic Review. J Am Geriatr Soc 2012; 60(8):1487-1492.

2. Haas VJ, Diniz MA, Aparecida R, Dantas S. Reprodutibilidade da versão brasileira adaptada da Edmonton Frail Scale para idosos residentes na comunidade. Rev. Latino-Am. Enfermagem 2013; 21(6):1330-1336.

3. Bandeen-Roche K, Xue Q, Ferrucci L, Walston J, Guralnik J, Chaves P, Zeger SL, Fried LP. Phenotype of frailty: characterization in the women's health and aging studies. The Journals of Gerontology Series A: Biological Sciences and Medical Sciences 2006; 61(3):262-266.

4. Fried LP, Tangen CM, Walston J, Newman AB, Hirsch C, Gottdiener J, Seeman T, Tracy R, Kop WJ, Burke G, McBurnie MA; Cardiovascular Health Study Collaborative Research Group. Frailty in older adults: evidence for a phenotype. J Gerontol A Biol Sci Med Sci 2001; 56(3):M146-M157.

5. Ravaglia G, Forti P, Lucicesare A, Pisacane N, Rietti E, Patterson C. Development of an easy prognostic score for frailty outcomes in the aged. Age Ageing. 2008; 37(2):161-166

6. Espinoza S, Walston JD. Frailty in older adults: insights and interventions. Cleveland Clinic Journal of Medicine 2005; 72(12):1105-1112.

7. Stuck AE, Walthert JM, Nikolaus T, Büla CJ, Hohmann C, Beck JC. Risk factors for functional status decline in community-living elderly people: a systematic literature review. Soc Sci Med 1999; (48):445-469.

8. Zoico E, Di Francesco V, Guralnik JM, Mazzali G, Bortolani A, Guariento S, Sergi G, Bosello O, Zamboni M. Physical disability and muscular strength in relation to obesity and different body composition indexes in a sample of healthy elderly women. International journal of obesity. 2004; 28(2):234-241.

9. Larrieu S, Peres K, Letenneur L, Berr C, Dartigues JF, Ritchie K, Février B, Alpérovitch A, Barberger-Gateau P. Relationship between body mass index and different domains of disability in older persons: the $3 \mathrm{C}$ study. Int J Obes Relat Metab Disord 2004; 28(12):1555-1560.

10. Chen H, Guo X. Obesity and functional disability in elderly Americans. J Am Geriatr Soc. 2008; 56(4):689694.

11. Ritchie CS, Locher JL, Roth DL, McVie T, Sawyer P, Allman R. Unintentional weight loss predicts decline in activities of daily living function and life-space mobility over 4 years among communitydwelling older adults. J Gerontol A Biol Sci Med Sci 2008; 63(1):67-75.

12. Romagnoni F, Zuliani G, Bollini C, Leoci V, Soattin L, Dotto S, Rizzotti P, Valerio G, Lotto D, Fellin R. Disability is associated with malnutrition in institutionalized elderly people. The I.R.A. Study. Istituto di Riposo per Anziani. Aging (Milan) 1999; 11(3):194-199.

13. Moretto MC, Alves RMDA, Neri AL, Guariento ME. Relação entre estado nutricional e fragilidade em idosos brasileiros. Rev Bras Clin Med. 2012; 10(4), 267271.
14. Tsai AC, Chang T-L. The effectiveness of BMI, calf circumference and mid-arm circumference in predicting subsequent mortality risk in elderly Taiwanese. $\mathrm{Br} J$ Nutr 2011; 105(2):275-281.

15. Izawa S, Enoki H, Hirakawa Y, Iwata M, Hasegawa J, Iguchi A, Kuzuya M. The longitudinal change in anthropometric measurements and the association with physical function decline in Japanese community-dwelling frail elderly. British journal of nutrition. 2010; 103(2):289-294.

16. Petroski EL. Antropometria: Técnicas e Padronizações. 2a ed. Porto Alegre: Palotti; 2003.

17. World Health Organization (WHO). Physical status: The use and interpretation of anthropometry. Geneva:WHO; 1995. (WHO technical report series, 854).

18. Alvarado BE, Zunzunegui MV, Béland F, Bamvita JM. Life Course Social and Health Conditions Linked to Frailty in Latin American Older Men and Women. J Gerontol A Biol Sci Med Sci 2008; 63(12):1399-1406.

19. American Academy of Family Physicians, American Dietetic Association, National Council On The Aging. Nutrition screening e intervention resources for healthcare professionals working with older adults. Nutrition Screening Initiative. Washington: American Dietetic Association; 2002.

20. Almeida OP, Almeida SA. Confiabilidade da versão brasileira da Escala de Depressão em Geriatria (GDS) versão reduzida. Arquivos de Neuro-Psiquiatria 1999; 57(2B):421-426.

21. Santos KT, Fernandes MH, Reis LA, Coqueiro RS, Rocha SV. Depressive symptoms and motor performance in the elderly: a population based study. Rev Bras Fisioter 2012; 16(4):295-300.

22. Craig CL, Marshall AL, Sjo“ Stro“ M, Bauman AE, Booth ML, Ainsworth BE, Pratt M, Ekelund U, Yngve A, Sallis JF, Oja P.International Physical Activity Questionnaire: 12-Country Reliability and Validity. Med. Sci. Sports Exerc 2003; 35(8):1381-1395.

23. Benedetti TRB, Mazo GZ, Barros MVG. Aplicação do questionário internacional de atividades físicas (IPAQ) para avaliação do nível de atividades físicas de mulheres idosas: validade concorrente e reprodutibilidade teste-reteste. Rev Bras Ciênc Mov 2004; 12(1):25-34.

24. Frisancho AR. New standards of weight and body composition by frame size and height for assessment of nutritional status of adults and the elderly. Am J Clin Nutr 1984; 40(4):808-819.

25. Harrison GG, Buskirk RE, Carter JEL, Johnston FE, Lohman TG, Pollock ML, Roche AF, ,Wilmore JH. Skinfold thicknesses. In: Lohman TG, Roche AF, Martorell R, editors. Anthropometric Standardization Reference Manual. Champaign: Human Kinetics; 1988. p. 55-80.

26. Callaway CW, Chumlea WC, Bouchard C, Himes JH, Lohman TG, Martin AD. Circumferences. In: Lohman TG, Roche AF, Martorell R, editors. Anthropometric standardization reference manual. Champaign: Human Kinetics Books. 1988: 44-45. 
27. Heymsfield SB, McManus C, Smith J, Stevens V, Nixon DW. Anthropometric measurement of muscle mass: revised equations for calculating bone-free arm muscle area. The American Journal of Clinical Nutrition 1982; 36(4):680-690.

28. Reis Junior WM, Carneiro JAO, Coqueiro RS, Santos KT, Fernandes MH. Pré-fragilidade e fragilidade de idosos residentes em município com baixo Índice de Desenvolvimento Humano. Rev. Latino-Am. Enfermagem 2014; 22(4):654-6152.

29. Katz S, Ford AB, Moskowitz RW, Jackson BA, Jaffe MW. Studies of illness in the aged. The index of ADL: a standardized measure of biological and psychosocial function. JAMA. 1963; 185(12):94-919.

30. Lawton MP, Brody EM. Assesment of older people: self-maintaining and instrumental activities of daily living. Gerontologist. 1969; 9(3):179-185.

31. Hoeymans N, Feskens EJ, van den Bos GA, Kromhout D. Measuring functional status: crosssectional and longitudinal associations between performance and self-report (Zuthen Elderly Study 1990-1993). J Clin Epidemiol. 1996; 49(10):1103-1110.

32. Berger MJ, Doherty TJ. Sarcopenia: prevalence, mechanisms, and functional consequences. Interdiscip Top Gerontol. 2010; 37(2):94-114.

33. Cruz-Jentoft AJ, Baeyens JP, Bauer JM, Boirie Y, Cederholm T, Landi F, Martin FC, Michel JP, Rolland Y, Schneider SM, Topinková E, Vandewoude M, Zamboni M; European Working Group on Sarcopenia in Older People. Sarcopenia: European consensus on definition and diagnosis. Report of the European Working Group on Sarcopenia in Older People. Age Ageing 2010; 39(4):412-423.

34. Pierine DT, Nicola M, Oliveira EP. Sarcopenia: alteracõos metabólicas e consequências no envelhecimento. Rev Bras Ciênc Mov 2009; 17(3):96-103.

35. Cawthon PM, Marshall LM, Michael Y, Dam TT, Ensrud KE, Barrett-Connor E, Orwoll ES, Osteoporotic Fractures in Men Research Group. Frailty in older men: prevalence, progression, and relationship with mortality. J Am Geriatr Soc. 2007; 55(8):1216-1223.

36. Almeida Silva N, Menezes TN, Melo RLP, Pedraza DF. Força de preensão manual e flexibilidade e suas relações com variáveis antropométricas em idosos. Revista da Associação Médica Brasileira. 2013; 59(2):128-135.

37. Blaum CS, Xue QL, Michelon E, Semba R, Fried LP. The association between obesity and the frailty syndrome in older women: the Women's Health and Aging Studies. J Am Geriatr Soc. 2005;53(6):927-934.

38. Enoki H, Kuzuya M, Masuda Y, Hirakawa Y, Iwata M, Hasegawa J, Iguchi A. Anthropometric measurements of mid-upper arm as a mortality predictor for community-dwelling Japanese elderly: The Nagoya Longitudinal Study of Frail Elderly (NLSFE). Clinical Nutrition 2007; 26(5):597-604.

39. Vellas BJ, Garry PJ, Guigoz Y. Nestlé Nutrition Services. Mini nutritional assessment (MNA):research and practice in the elderly. Nestle Nutrition Workshops Series Clinical \& Performance Programme 1999; 1: -12.
40. Reid KF, Naumova EN, Carabello RJ, Phillips EM, Fielding RA. Lower extremity muscle mass predicts functional performance inmobility-limited elders. $J$ Nutr Health Aging 2008; 12(7):493-498.

41. Menezes TN, Brito MT, Araújo TBP, Silva CCM, Nolasco RRN, Fischer MATS. Perfil antropométrico dos idosos residentes em Campina Grande-PB. Rev. Bras. Geriatr. Gerontol 2013; 16(1):19-27.

42. Rebelatto JR, Castro AP, Chan A. Quedas em idosos institucionalizados: características gerais, fatores determinantes e relações com a força de preensão manual. Acta Ortop Bras 2007; 15(3):151-154.

43. Jones DM, Song X, Rockwood K. Operationalizing a frailty index from a standardized comprehensive geriatric assessment. J Am Geriatr Soc 2004; 52(11):19291933.

Artigo apresentado em 14/12/2015

Aprovado em 04/05/2016

Versão final apresentada em 06/05/2016 
